\title{
Hydrodynamic modelling of protein conformation in solution: ELLIPS and HYDRO
}

\author{
José García de la Torre • Stephen E. Harding
}

Received: 11 December 2012 / Accepted: 22 January 2013 / Published online: 20 February 2013

(C) The Author(s) 2013. This article is published with open access at Springerlink.com

\begin{abstract}
The last three decades has seen some important advances in our ability to represent the conformation of proteins in solution on the basis of hydrodynamic measurements. Advances in theoretical modeling capabilities have been matched by commensurate advances in the precision of hydrodynamic measurements. We consider the advances in whole-body (simple ellipsoid-based) modeling — still useful for providing an overall idea of molecular shape, particularly for those systems where only a limited amount of data is available - and outline the ELLIPS suite of algorithms which facilitates the use of this approach. We then focus on bead modeling strategies, particularly the surface or shell-bead approaches and the HYDRO suite of algorithms. We demonstrate how these are providing great insights into complex issues such as the conformation of immunoglobulins and other multi-domain complexes.
\end{abstract}

Keywords Solution conformation · Whole-body models · Bead and shell models

\section{Introduction}

There are two approaches to the hydrodynamic modelling of proteins. The simplest is "whole-body modelling" using ellipsoids which involves the exact hydrodynamic equations

Special Issue: Protein-Protein and Protein-Ligand Interactions in Dilute and Crowded Solution Conditions. In Honor of Allen Minton's 70th Birthday

J. García de la Torre

Departamento de Quimica Fisica, Universidad de Murcia,

Regional Campus Mare Nostrum,

30071 Murcia, Spain

S. E. Harding $(\square)$

School of Biosciences, University of Nottingham,

Sutton Bonington,

LE12 5RD England, UK

e-mail: steve.harding@nottingham.ac.uk linking hydrodynamic measurements with the axial ratios describing the ellipsoidal shape. This approach which has been used since Perrin $(1934,1936)$ and Simha (1940) provided relationships linking the axial ratio for both prolate and oblate ellipsoids of revolution (ellipsoids with two equal axes) with the frictional properties (rotational and translational) and intrinsic viscosity, respectively. Progress was made in the 1940s-1950s in dealing with the "hydration problem", namely the recognition that the frictional ratio and intrinsic viscosity had contributions not only from asymmetry but also the volume of the protein, "swollen" by the time-averaged interaction with the surrounding solvent. Graphical (Mehl et al. 1940; Oncley 1940), and analytical solutions for evaluating the axial ratio and hydration were provided (Scheraga and Mandelkern 1953). Intriguingly, Saito (1951) had questioned Simha's derivation on the basis of an apparently erroneous assumption (macromolecules rotating with zero angular velocity in viscous flow rather than rotating at the same angular velocity as the surrounding liquid), whilst arriving at the same relationship himself. Simha's derivation was later shown to have reached the correct formula on the basis of an apparent cancellation of errors (Harding et al. 1982). Further progress was made with the removal of the restriction of two equal axes and the introduction of the general triaxial ellipsoid in the 1980s by combination of three different types of hydrodynamic measurement (see, e.g., Harding 1987). Calculation of the relevant parameters required solution of elliptic integrals: for ellipsoids of revolution, these integrals could be solved analytically using quadrature-although relationships were relatively complex, tabular or graphical values as a function of axial ratio became available along with simpler-to-use approximate formulae. For general ellipsoids, numerical solution of the integrals was needed using high-speed computers. Attention then moved to the production of easy-to-use algorithms, an endeavour which led to the ELLIPS algorithms now downloadable on www.nottingham.ac.uk/ncmh. 
Whole-body modelling may be insufficient when finer structural details are being investigated, and it has proved inappropriate for representing the conformation of many classes of protein, particularly non-globular protein shapes, and multi-subunit structures, as is the case of antibodies and a variety of macromolecular complexes. It was, indeed, the lack of adequacy of ellipsoidal models for such cases that prompted the pioneering work of Bloomfield and coworkers in the 1960s (Bloomfield et al. 1967), who proposed application of the bead model concept, initially developed in polymer physics for simple, chain-like or rod-like structures, to model the peculiar shapes of biomacromolecules. The concept evolved over the years (see, e.g., Garcia de la Torre and Bloomfied 1981; Carrasco and Garcia de la Torre 1999) by implementing new theoretical advances into computational algorithms that made use of the continuously increasing computing power. After the publication of the first publicdomain bead-modeling program HYDRO (García de la Torre et al. 1994), a suite of software has been developed and made available to the scientific community (http://leonardo. inf.um.es/macromol/). This methodology can now be applied to a variety of complex situations quite simply and with considerably reduced computing requirements.

In this short review, we consider aspects of the ELLIPS and HYDRO suites of algorithms for whole-body and bead model representations of conformation in solution. For fuller details, the researcher is referred to the respective websites.

\section{Whole body models - the ELLIPS suite of algorithms}

With whole-body modelling we make no assumptions concerning starting estimates for the structure-other than assuming the molecule is rigid - and calculate the shape directly from the measurement of one or more hydrodynamic parameters. The shape model is a smooth whole regular structure - namely an ellipsoid characterised by three perpendicular semi-axes $a, b, c$, with $a \geq b \geq c$, and two independent axial ratios (conventionally $a / b, b / c$ ). The common limiting forms are the prolate (with semi-axes $a, b, b$ ) and oblate $(a, a, b)$ "ellipsoids of revolution" described by a single axial ratio $a / b$, with $a>b$ in both cases.

The ELLIPS algorithms were developed for performing this type of modelling, initially for use on mainframe computers (Harding 1983), then for MS-DOS (Harding et al. 1997), and finally for the Windows platform (Harding et al. 2005). It has been periodically updated for alterations in Window platforms on www.nottingham.ac.uk/ncmh, the latest being for Windows XP or Windows 7.

Table 1 gives a summary of what these do. Obviously, many classes of molecule cannot be reasonably represented by a smooth symmetrical shape-antibodies are a good example - this type of whole-body modelling is not applicable and bead-approaches need to be employed. Even here, however, ellipsoidal representations of the major domains (Fab, Fc) have helped in the bead modelling of the intact assembly (Carrasco et al. 1999, 2001; Longman et al. 2003; Lu et al. 2006, 2007).

\section{Universal shape functions-ELLIPSPRIME}

The ELLIPS algorithms work with "universal shape" parameters-i.e. parameters which can be described by a unique function of shape and not of size. The simplest of these is the Einstein-Simha shape function $v$, which is measurable from the intrinsic viscosity of a protein and has a value $\geq 2.5$ (the limiting value being for a sphere). Another is the Perrin function $\mathrm{P}$, which is measurable from the sedimentation coefficient or translational diffusion coefficient of a protein and has a value $\geq 1$. All these universal shape functions have been worked out in terms of the axial ratio $(a / b)$ for ellipsoids of revolution and also the two axial ratios $(a / b, b / c)$ for general ellipsoids. The relations of all these to $(a / b)$ or $(a / b, b / c)$ are given in Harding (1995). To measure these shape functions experimentally, many-including $v$ and $\mathrm{P}$-require knowledge of the hydration $\delta$ (mass in $\mathrm{g}$ of $\mathrm{H}_{2} \mathrm{O}$ associated per $\mathrm{g}$ of dry macromolecule) or hydrated volume $V(\mathrm{ml})$ of the particle, though the others do not. Hydration is a dynamic process, and so $\delta$ and $V$ represent time-averaged values. It is wrong, however, to assume that because of the dynamic nature of hydration it has no affect on hydrodynamic properties. The residence time for water/solvent in the so-called hydration layer(s) has been shown to be different from that in bulk water (Denisov and Halle 1996). The particle volume $V$ is often presented in two equivalent forms:

$V=\mathrm{v}_{\mathrm{s}} \cdot M / N_{A}$

where $M$ is the molecular weight or molar mass $(\mathrm{g} / \mathrm{mol})$ and $N_{A}$ is Avogadro's number $\left(6.02205 \times 10^{23} \mathrm{~mol}^{-1}\right)$, and $\mathrm{v}_{\mathrm{s}}$ is the specific volume $(\mathrm{ml} / \mathrm{g})$ of the hydrated macromolecule (volume occupied by the hydrated macromolecule per unit mass of dry macromolecule) or

$V=\left(\overline{\mathrm{v}}+\delta / \rho_{\mathrm{o}}\right) \cdot M / N_{A}$

where $\bar{v}$ is the partial specific volume $(\mathrm{ml} / \mathrm{g})$.

Examples of universal shape functions requiring knowledge of $\delta$ or $V$ for their experimental measurement include the viscosity increment, $v$

$v=[\eta] M /\left(N_{A} V\right)$ 
Table 1 The ELLIPS suite of whole-body modeling algorithms

\begin{tabular}{|c|c|c|c|}
\hline Routine & Language & Model & Purpose \\
\hline ELLIPS1 & QUICKBASIC & Ellipsoid of Revolution & $\begin{array}{l}\text { Prediction of axial ratio }(a / b) \text { from any user } \\
\text { specified shape function }\end{array}$ \\
\hline ELLIPS2 & FORTRAN & $\begin{array}{l}\text { General Triaxial } \\
\text { Ellipsoid }\end{array}$ & $\begin{array}{l}\text { Evaluates all the hydrodynamic shape functions } \\
\text { from user specified }(a, b, c) \text { or }(a / b, b / c)\end{array}$ \\
\hline ELLIPS3 & FORTRAN & $\begin{array}{l}\text { General Triaxial } \\
\text { Ellipsoid }\end{array}$ & $\begin{array}{l}\text { Evaluates }(a / b, b / c) \text { from combinations of } \\
\text { hydration independent shape functions }\end{array}$ \\
\hline ELLIPS4 & FORTRAN & $\begin{array}{l}\text { General Triaxial } \\
\text { Ellipsoid }\end{array}$ & $\begin{array}{l}\text { Evaluates }(a / b, b / c) \text { from electro-optic decay } \\
\text { combined with other data }\end{array}$ \\
\hline
\end{tabular}

and the Perrin function, $P$

$P=\left(f / f_{\mathrm{o}}\right) /\left\{1+\delta /\left(\overline{\mathrm{v}} \rho_{\mathrm{o}}\right)\right\}^{1 / 3}$

where $\left(f / f_{\mathrm{o}}\right.$ ), the frictional ratio (Tanford 1961) which can be related to the sedimentation coefficient $s^{\mathrm{o}}{ }_{20, \mathrm{w}}$ or diffusion coefficient

Another popular parameter is the reduced excluded volume (Rallison and Harding 1985), $\mathrm{u}_{\mathrm{red}}$

$u_{\text {red }}=u / V=\left\{2 B M^{2}-\mathrm{f}(\mathrm{Z}, \mathrm{I})\right\} /\left(N_{A} V\right)$

where $u$ is the excluded volume $(\mathrm{ml}), B$ is the second thermodynamic (or "osmotic pressure") virial coefficient, from osmotic pressure, light scattering or sedimentation equilibrium measurements, and $\mathrm{f}(Z, I)$ is a function of the valency $\mathrm{Z}$ of the macromolecule and ionic strength I of the solution. $u_{\text {red }}=8$ for a sphere (Tanford 1961).

There are also a plethora of shape functions relating to rotational frictional behaviour (fluorescence anisotropy decay and electric birefringence decay) described in Harding (1983) and Harding et al., (1997, 2005). In addition there are also universal shape functions that do not require knowledge of $\delta$ or $V$ for their experimental measurement. These are obtained by combining two hydrodynamic parameters. The classical example is the Scheraga-Mandelkern $\beta$ parameter (from combination of $v$ with $\mathrm{P}$ ) - although this is a very insensitive function of shape. Another and much more useful example is the Pi function (Harding 1981) from the combination of intrinsic viscosity measurement with measurement of the 2nd thermodynamic virial coefficient:

$\Pi=\{2 B M /[\eta]\}-\left\{Z^{2} / 2 I M[\eta]\right\}=u_{\text {red }} / \nu$

Other examples involve combination of either $v$ or $\mathrm{P}$ with rotational frictional-based functions such as the harmonic mean rotational relaxation time. To assist with the calculation of the Universal parameters such as $v, \mathrm{P}$ and $u_{\text {red }}$, and $\Pi$ from the relevant experimental parameters, an Excel spreadsheet routine ELLIPSPRIME has been set up specifically for this purpose.

\section{Ellipsoids of revolution: ELLIPS1}

ELLIPS1 is based on simple ellipsoid of revolution models (where two of the three axes of the ellipsoid are fixed equal to each other); if the user enters a value for a shape function from sedimentation or other types of hydrodynamic measurement, it will return a value for the axial ratio of the ellipsoid. The question an experimenter wishes to address usually is not "what is the shape function for a specified value of the axial ratio $a / b$ ?" but rather "what is the axial ratio $a / b$ for my macromolecule specified by my (universal) shape function which I have experimentally measured?". Although there are exact analytical formulae linking each shape function with $a / b$, the reverse is not true: inversion is analytically impossible. The QUICKBASIC algorithm ELLIPS1 uses the polynomial-based inversion procedure of Harding et al. (1997) to give $a / b$ versus the various universal shape functions to an acceptable degree of accuracy (i.e. to better than the precision of the measurement, which is normally no better than a few percent). Figure 1 gives some recent published examples.

\section{Evaluation of hydrodynamic properties from the axial dimensions of a protein: ELLIPS2}

ELLIPS2 evaluates exactly a complete set of universal shape functions for user-specified values of the axial ratios of a protein. All the user has to do is enter the two axial ratios $(a / b, b / c)$ for the protein from the axial dimensions of the molecule (e.g. from x-ray crystallography). Since prolate and oblate ellipsoids of revolution are two limiting forms of the general ellipsoid, the program will also of course evaluate the set of parameters for these two situation, as well as for (rigid) tapes $(a>>b>>c)$. An example of the method has been evaluation of the hydrodynamic parameters for tri-axial ellipsoid models for IgG Fab and Fc domains, using the crystallographic dimensions for these structures (Fig. 2). For example, $\mathrm{P}$ values were evaluated for this and 
a

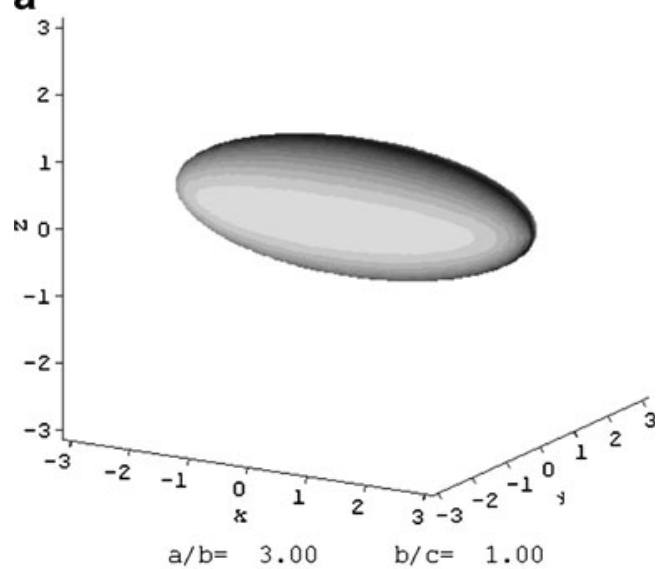

b

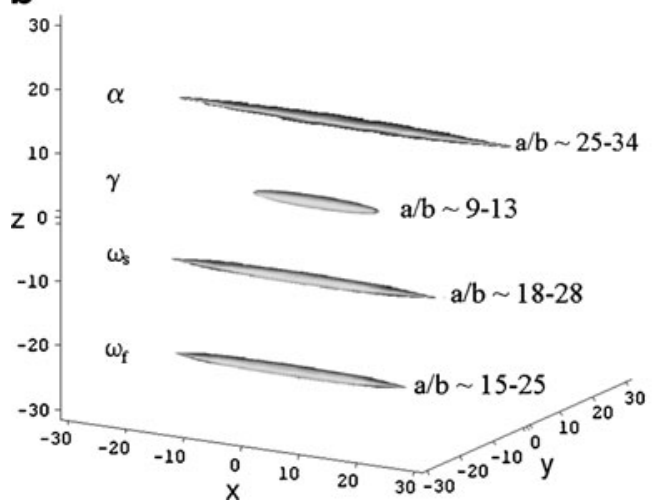

Fig. 1 ELLIPS1 representations for a the tetanus toxoid protein, used in glycoconjugate vaccines (adapted from Abdelhameed et al. 2012) and $\mathbf{b}$ wheat protein gliadins $\alpha, \beta, \omega_{\mathrm{f}}$ and $\omega_{\mathrm{s}}$ (Ang et al. 2010)

compared with the corresponding bead-shell model representations of the surfaces, validating the latter for subsequent use in construction of models for the intact antibody structure.

\section{Evaluation tri-axial dimensions from hydrodynamic parameters ELLIPS3 and ELLIPS4}

ELLIPS3 and 4 perform the reverse of ELLIPS2 by evaluating the tri-axial shape of a macromolecule $(a / b, b / c)$ from hydrodynamic measurements. At least three experimental parameters are required since there are 3 unknowns $-a / b$, $b / c$ and the hydration $\delta$. For example, ELLIPS3 has been set up to allow the evaluation of $(a / b, b / c)$ from a combination of measurements of the intrinsic viscosity, the second thermodynamic virial coefficient and the radius of gyration, or from a combination of the intrinsic viscosity with the harmonic mean rotational relaxation time (from steady state fluorescence depolarisation experiments) and sedimentation data. For example, it has been successfully applied to the a

ELLIPS2 Output Data

$(\mathrm{a} / \mathrm{b}, \mathrm{b} / \mathrm{c})=1.6000 \quad 1.4200$

Viscosity increment, $\mathrm{nu}=2.9120$

Perrin function, $P=1.0457$

Corresponding hydration independent functions:

Walesvan Holde, $\mathrm{R}=1.4721$

Scheraga-Mandelkern, $10^{\star \star}$-6xbeta $=2.1247$

Functions based on the 2nd virial coefficient:

Reduced excluded volume, u_red 9.1442

Corresponding hydration independent function:

Pi function $=3.1401$

$\mathrm{G}$ function (from radius of gyration) $=0.7491$

Reduced electro -optic decay constants:

Theta+ $=0.1654$

Theta $=0.1134$

Corresponding hydration independent functions:

Delta $+=2.8907$

Delta $=1.9806$

Gamma+ = 1.6965

Gamma $=1.1624$

Harmonic mean rotational relaxational time ratio: tau $\mathrm{h} / \mathrm{tau} 0=1.1956$

Corresponding hydration-independent functions:

PSI (Squire -Himmel) function $=0.9853$

LAMBDA $=2.4356$

Fluorescence anisotropy relaxation time ratios:

tau_1/tau_0 = 1.0075

tau_2/tau_0 $=1.3083$

tau $3 / \operatorname{tau} 0=1.3292$

tau $4 /$ tau $0=1.4703$

tau_5/tau_0 $=1.0074$

Corresponding hydration independent functions:

lambda_1 = 2.8903

lambda $2=2.2259$

lambda_3 $=2.1908$

lambda $4=1.9806$

lambda $\_5=2.8907$

b

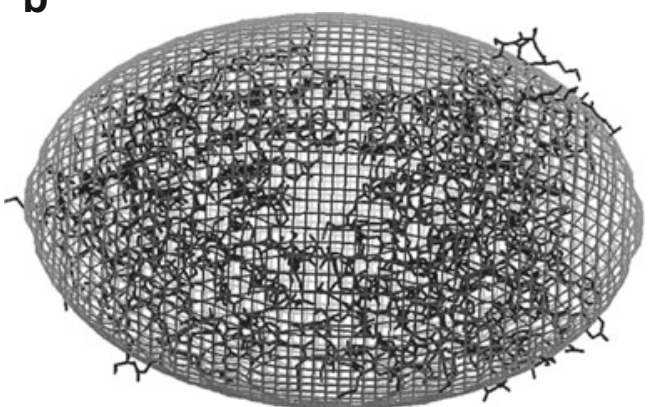

Fig. 2 a ELLIPS2 output for IgG antibody domain B72.3c Fab' (see Carrasco et al. 2001). b The axial ratios of $(a / b, b / c)=1.60,1.42)$ used for this example were obtained from the crystal structure of Brady et al. (1992) using the algorithm of Taylor et al. (1983), which fits a surface ellipsoid to crystallographic atomic co-ordinate data from the protein data bank (PDB)

study of myosin rods and the study of the mode of dimerisation of the neural protein neurophysin. ELLIPS3 uses as its basis the function calculation routine of ELLIPS2 except 
that a whole array of such values are evaluated in the $(a / b$, $b / c$ ) plane (a matrix of $40 \times 40$ values). A contour plotting routine (RGCNTS from the Simpleplot Library) interpolates between these matrix points, thereby effecting a combination of line solutions to yield a unique solution with allowance for experimental error, of $(a / b, b / c)$ for a protein. ELLIPS4 is similar but involves a combination of intrinsic viscosity and sedimentation data with electric birefringence decay data, using a constrained fit procedure for extraction of the decay constants.

\section{Bead models - the HYDRO suite of algorithms and the concept of equivalent radii}

As mentioned in the Introduction, after the first release of the HYDRO computer program for hydrodynamics of bead models (Garcia de la Torre et al. 1994), the collection of computer programs has been considerably expanded. Hereafter, we shall give some details for only those tools that are most relevant for the study of protein hydrodynamics, particularly the programs HYDROPRO, HYDRONMR and HYDROSUB. It is convenient to mention here another advance, which consists of an interface between the results of those programs and experimental data, in order to search for conformations that would fit a set of data for several proteins. For such a purpose, we have found it convenient to employ the solution properties in the form of equivalent radii (Ortega and Garcia de la Torre 2007). For a given value of some property, the equivalent radius of the particle is the radius that a spherical particle would have in order to reproduce that value. For instance, the equivalent radius for translational hydrodynamics, sometimes called Stokes radius, is defined as $a_{\mathrm{T}}=f / 6 \pi \eta_{0}$ where $f$ is the translational friction coefficient that can be determined from translational diffusion or sedimentation. Examples of other equivalent radii are that corresponding to intrinsic viscosity, $a_{\mathrm{I}}=(3$ $[\eta] M / 10 \pi)^{1 / 3}$, and that from the radius of gyration $a_{\mathrm{G}}=$ $(5 / 3)^{1 / 2} R_{\mathrm{g}}$ The adequacy of an assumed structure to fit a set of experimental values for several properties can be judged in terms of the square deviations or the calculated and experimental equivalent radii. A convenient measure of the degree of fit is given by the quantity

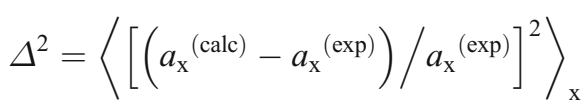

where $\langle\ldots\rangle_{\mathrm{X}}$ indicates the mean over the various properties that are available. Note that $100 \Delta$ is the typical percent relative deviation of the calculated radii from the experimental ones. In some instances, the structure is characterised in terms of some parameters, so that $\Delta\left(p_{1}, p_{1}, \ldots\right)$ would be a function of those parameters, whose optimum values, defining the best fitting structure, are those which minimise $\Delta$. The HYDRO suite contains an ancillary program, HYDROFIT (Ortega et al. 2011b), intended to carry out such a kind of structural optimisation from the results produced by HYDRO, HYDROPRO, HYDROSUB, etc. (Table 2).

\section{Calculation of solution properties of rigid proteins from their detailed molecular structure}

Simple, single-valued quantities like the hydrodynamic coefficients and the radius of gyration are low-resolution properties, and one cannot hope to extract from them highly detailed structural information. On the other hand, highresolution techniques like diffraction and NMR provide detailed, even atomic-level, structures, although at the cost of complex instrumentation and data handling. Historically, these techniques came after many decades during which the structural elucidation of proteins was mainly based on hydrodynamic properties, analysed in terms of primitive ellipsoidal models. However, it was realized that, because of the complexity of their determination, such structures should be validated against experimental data of readily accessible properties of the protein in solution.

This purpose motivated the development of a methodology for predicting solution properties from high-resolution structures with even atomic-level detail. A pioneering

Table 2 The HYDRO suite of modelling programs

\begin{tabular}{lll}
\hline Program & Starting structure/information & Results \\
\hline HYDRO & $\begin{array}{l}\text { User-constructed model } \\
\text { Spherical beads: coordinates and radii }\end{array}$ & A, B \\
HYDROPRO & $\begin{array}{l}\text { Atomic- or residue-level structure. } \\
\text { (PDB-formatted list of coordinates) }\end{array}$ & A, B \\
HYDRONMR & $\begin{array}{l}\text { Atomic- level structure. } \\
\text { (PDB-formatted list of coordinates) }\end{array}$ & C \\
HYDROMIC & $\begin{array}{l}\text { 3D density map from electron } \\
\text { microscopy, several formats } \\
\text { "Dummy atoms" model from small-angle } \\
\text { HYDROSAS }\end{array}$ & A \\
HYDROSUB & $\begin{array}{l}\text { User-constructed model } \\
\text { Ellipsoids, cylinders: size, position and } \\
\text { orientation }\end{array}$ & A, B \\
HYDFIT & $\begin{array}{l}\text { Experimental data } \\
\text { Output from any of the HYDROxxx } \\
\text { programs }\end{array}$ & D \\
\hline
\end{tabular}

$A$ Hydrodynamic coefficients (sedimentation, diffusion, rotational times, intrinsic viscosity), radius of gyration, covolume

$B$ Angular dependence of scattering intensities and distribution of distances

$C$ NMR relaxation times

$D$ Selection of an optimum, best-fitting structure 
attempt, when x-ray diffraction structures were still scarce, was that by Teller et al. (1976). The availability of developments in hydrodynamic computations, implemented in the computer program HYDRO (Garcia de la Torre et al. 1994), prompted further advances in the application to proteins; among many others, the work of Byron (1997) for atomiclevel models is particularly remarkable. Some aspects in hydrodynamic modelling that were deficient by then were improved later on (Carrasco and Garcia de la Torre 1999), providing the framework for a methodology specific for rigid proteins, implemented in the program HYDROPRO (Garcia de la Torre et al. 2000), which allowed the calculation of solution properties from a set of atomic coordinates in PDB format. Since then, some alternative procedures have been proposed (Rai et al. 2005; Aragon and Hahn 2006; Brookes et al. 2010), but HYDROPRO has been, by far, the most frequently used tool for this purpose. Very recently (Ortega et al. 2011a, b), this program has been fully refurbished, with inclusion of new modelling strategies and improvements in computational aspects. Hereafter, we summarise the main features of the present methodology

In the original HYDROPRO procedure (Garcia de la Torre et al. 2000), an atomic-level structure, as specified in a set of $N_{\text {at }}$ atomic coordinates, was the origin of a primary hydrodynamic model (PHM) in which each nonhydrogen atom is replaced by a spherical element, beads of radius $a_{\mathrm{at}}$ which should be even larger than van der Waals radii in order to account for hydration, so that beads in the PHM overlap appreciably. In order to avoid some hydrodynamic problems regarding bead overlapping, the PHM was in turn replaced by a shell model, in which a number of hydrodynamic centres, $N$, of non-overlapping "minibeads" of radius $\sigma$ cover the surface of the PHM. Properties are calculated for several, increasing values of $\mathrm{N}$, up to about 2,000 , with decreasing sigma, and the results extrapolated to $N \rightarrow \infty$. The hydrodynamic calculations require handling a $3 N \times 3 N$ matrix (which for the finest shell model would be $6,000 \times 60,00$ ), with computing time proportional to $N^{3}$.

The present, state-of-the-art HYDROPRO methodology includes important improvements, particularly in efficiency achieved by techniques of high-performance computing, and new modelling features. One of them is the possibility of predicting the solution properties from a lower resolution, non-atomic, residue-level model of the protein, specified by a list of coordinates of $\mathrm{C}^{\alpha}$ atoms. Another refers to the hydrodynamic treatment of bead overlapping, that has allowed the possibility of making the hydrodynamic calculation directly from the primary hydrodynamic model (PHM) - which in the case of the residue-level model would contain a number of elements equal to the number of amino acid residues, $N_{\text {res }}$. Computing time would be then proportional to $N_{\text {res }}{ }^{3}$, with an evident computational advantage over the shell calculations for proteins of less than, say,
1,000 residues. Thus, there are now various computational strategies possibilities:

(a) "Atomic/PHM $\rightarrow$ Shell". An atomic PHM with overlapping beads of $a=2.9 \AA$, built from atomic coordinates, is in the computation replaced by a shell model of up to $N \cong 20,00$ minibeads

(b) "Residue/PHM $\rightarrow$ Shell". A residue-level PHM with overlapping beads of $a=5.0 \AA$, built from $\mathrm{C} \alpha$ coordinates, is in the computation replaced by a shell model of up to $N \cong 20,00$ minibeads

(c) "Residue/PHM". A residue-level PHM with overlapping beads of $a=6.1 \AA$, built from $\mathrm{C} \alpha$ coordinates, is directly used in the computation, which involves $N_{\text {res }}$ beads

Note that all the methods require a PHM, so that the primary parameter is the radius, $a$, of the (overlapping) beads in the PHM. As in the primitive version of HYDROPRO (Garcia de la Torre et al. 2000), this parameter has been adjusted so as to reproduce experimental data for small- and medium-sized proteins, with the improvement in the new version of making the parameterisation with an extensive set of various properties (translational coefficients, intrinsic viscosity, rotational relaxation) for over 70 proteins and of over 150 experimental data. For the global fit of such large and complex set, we employed the procedure described above based on the optimisation of equivalent radii. The results are displayed in Table 3.

It seems clear from the summary in Table 3 and the examples in Fig. 3 that our procedures provide highly accurate predictions for the radius of gyration, Stokes radius and other hydrodynamic radii; the deviation is in most cases within the range 3-6\%, and, considering that this includes the uncertainty of the experimental data themselves, then the errors introduced by the modelling and computational procedures are, indeed, quite small.

It is also noteworthy that models derived from residuelevel structures provide predictions of the same quality as those from atomic models. This possibility seems of practical importance, as it extends the utility of HYDROPRO to cases where only lower-resolution structures are available. Another important feature is that the hydrodynamic calculation made directly from the residue-level PHM in the Residue/PHM method, with $N_{\text {res }}$ hydrodynamic elements, performs nearly as well as the Residue/PHM $\rightarrow$ Shell and Residue/PHM $\rightarrow$ Shell procedures, both with a number of elements $N$ up to 2,000 . The computing time for the shell methods is not long (less than 3 min on typical, inexpensive personal computers); nonetheless, in cases where CPU consumption may be important - as in high-throughput structural search software in which HYDROPRO may be implemented (Bernado and Blackledge 2009; Cho et al. 2009; Krzeminski et al. 2013) —with an unappreciable 
Table 3 Best-fitting values of the bead size, $a$, in the PHM of proteins for the several computing strategies, and typical (mean) percent deviation, \% dif., found for translational properties, radius of gyration, intrinsic viscosity and relaxation time. Figure 3 presents numerical values for some specific, well-known proteins of which more than one property are available

\begin{tabular}{|c|c|c|c|c|c|c|}
\hline Method & $a, \AA$ (in PHM) & $\begin{array}{l}\% \text { dif. } a_{\mathrm{X}}, \\
\text { (from all prop.) }\end{array}$ & $\begin{array}{l}\% \text { dif. } a_{\mathrm{G}}, \\
\text { (from } R_{\mathrm{g}} \text { ) }\end{array}$ & $\begin{array}{l}\% \text { dif. } a_{\mathrm{T}} \\
\text { (from } D \text { or } s \text { ) }\end{array}$ & $\begin{array}{l}\% \text { dif. } a_{\mathrm{I}}, \\
\text { (from }[\eta] \text { ) }\end{array}$ & $\begin{array}{l}\% \text { dif. } a_{\mathrm{R}}, \\
\text { from } \tau \text { ) }\end{array}$ \\
\hline Atomic/PHM $\rightarrow$ Shell & 2.9 & 4.5 & 3.9 & 4.7 & 3.8 & 5.3 \\
\hline Residue/PHM $\rightarrow$ Shell & 4.8 & 4.4 & 4.0 & 4.7 & 3.5 & 5.4 \\
\hline Residue/PHM & 6.1 & 4.8 & 6.7 & 5.0 & 3.9 & 5.0 \\
\hline
\end{tabular}

sacrifice in accuracy, our new non-shell alternative may be much more efficient for small- and medium-sized proteins (with less than, say, 1,000 residues) than the shell methodologies employed in our previous HYDROPRO (Garcia de la Torre et al. 2000) and other analogous procedures (Aragon and Hahn 2006).

On the other hand, for large proteins and macromolecular complexes, the shell model procedures, with a fixed $\mathrm{N}$ and computing time independent on their size, is computationally advantageous. Methods in which the number of elements in the hydrodynamic model is related to the protein size, like our Residue/PHM or others (Brookes et al. 2010), may result in becoming too expensive or may even be not feasible for such cases. However, with the Atomic/PHM $\rightarrow$ Shell or Residue/PHM $\rightarrow$ Shell procedures, one can make hydrodynamic calculations with the same computing time, and a similarly good accuracy, for GroEL, or the 70S ribosomal particle, as for a protein as small as BPTI. Figure 4 presents a summary illustrating the performance of the three approaches for proteins across a wide range of molecular weight.

\section{NMR relaxation: HYDRONMR}

Single-valued properties, like the sedimentation and diffusion coefficient, the intrinsic viscosity or the radius of gyration reflect, in a single numerical value, the overall conformation of the protein, i.e. its size and shape in the case of rigid molecules.

\begin{tabular}{|c|c|c|c|c|c|}
\hline Protein & $\begin{array}{l}\text { No. } \\
\text { res., } \\
\mathrm{Nr}\end{array}$ & $a_{x}$ & $\begin{array}{c}\text { Atomic } \\
\text { Shell } \\
N=2000\end{array}$ & $\begin{array}{c}\text { Residue } \\
\text { Shell } \\
N=\mathbf{2 0 0 0}\end{array}$ & $\begin{array}{r}\text { Residue } \\
\text { Primary } \\
N=N r\end{array}$ \\
\hline BPTI & 56 & $a_{T}$ & -5.8 & 8.5 & 6.5 \\
\hline (4PTI) & & $a_{R}$ & -1.8 & 0.9 & 0.0 \\
\hline \multirow{4}{*}{$\begin{array}{l}\text { Lysozyme } \\
\text { (6LYZ) }\end{array}$} & \multirow{4}{*}{129} & $a_{G}$ & -2.5 & -4.5 & 6.4 \\
\hline & & $a_{T}$ & 0.9 & 0.9 & 0.0 \\
\hline & & $a_{1}$ & -3.1 & -3.1 & -3.8 \\
\hline & & $a_{R}$ & 1.1 & 1.1 & 0.3 \\
\hline \multirow{3}{*}{$\begin{array}{l}\text { Chymotrypsinogen } \\
\text { (2CGA) }\end{array}$} & \multirow{3}{*}{245} & $a_{G}$ & 3.3 & 0.6 & 9.9 \\
\hline & & $a_{T}$ & -2.1 & -2.7 & -4.1 \\
\hline & & $a_{1}$ & -4.8 & -5.2 & -6.2 \\
\hline \multirow{3}{*}{$\begin{array}{l}\text { Beta-Lactoglobulin } \\
\text { (1BEB) }\end{array}$} & \multirow{3}{*}{324} & $\bar{a}$ & -4.2 & -3.2 & 1.9 \\
\hline & & $a_{T}$ & -1.4 & 1.6 & 0.3 \\
\hline & & $a_{R}$ & -2.4 & 0.9 & 0.1 \\
\hline \multirow{2}{*}{$\begin{array}{l}\text { Oxyhaemoglobin } \\
(1 \mathrm{HHO})\end{array}$} & \multirow[t]{2}{*}{574} & $a_{T}$ & -0.9 & -0.4 & -0.9 \\
\hline & & $a_{1}$ & -5.9 & -5.4 & -5.2 \\
\hline \multirow{3}{*}{$\begin{array}{l}\text { Citrate synthase } \\
\text { (1CTS) }\end{array}$} & \multirow{3}{*}{874} & $a_{G}$ & 0.3 & 0.0 & 5.2 \\
\hline & & $a_{T}$ & -3.0 & -1.4 & -2.1 \\
\hline & & $a_{1}$ & 3.1 & 4.6 & 4.5 \\
\hline \multirow{2}{*}{$\begin{array}{c}\text { Aldolase } \\
(1 \mathrm{ADO})\end{array}$} & \multirow[t]{2}{*}{1452} & $a_{T}$ & -0.2 & 2.6 & -0.2 \\
\hline & & $a_{1}$ & -3.6 & -2.7 & -2.8 \\
\hline Urease (3LA4) & 4996 & $a_{T}$ & -3.6 & -2.8 & -6.9 \\
\hline \multirow{2}{*}{$\begin{array}{c}\text { GroEL } \\
(2 \mathrm{CGT})\end{array}$} & \multirow[t]{2}{*}{7273} & $a_{G}$ & 1.3 & 3.9 & 3.0 \\
\hline & & $a_{T}$ & -0.4 & 3.4 & -0.4 \\
\hline \multirow{3}{*}{$\begin{array}{c}\lg \mathrm{M} \\
(2 \mathrm{RCJ})\end{array}$} & \multirow{3}{*}{7514} & $a_{G}$ & - & -4.7 & -4.4 \\
\hline & & $a_{T}$ & - & -5.2 & -7.1 \\
\hline & & $a_{1}$ & - & -0.7 & -0.9 \\
\hline \multirow{2}{*}{$\begin{array}{l}\text { Ribosome 70S } \\
\text { (1VSA \& 2OW8) }\end{array}$} & \multirow[t]{2}{*}{10428} & $a_{G}$ & 6.4 & 4.3 & 6.4 \\
\hline & & $a_{T}$ & -1.7 & -3.4 & -0.8 \\
\hline
\end{tabular}

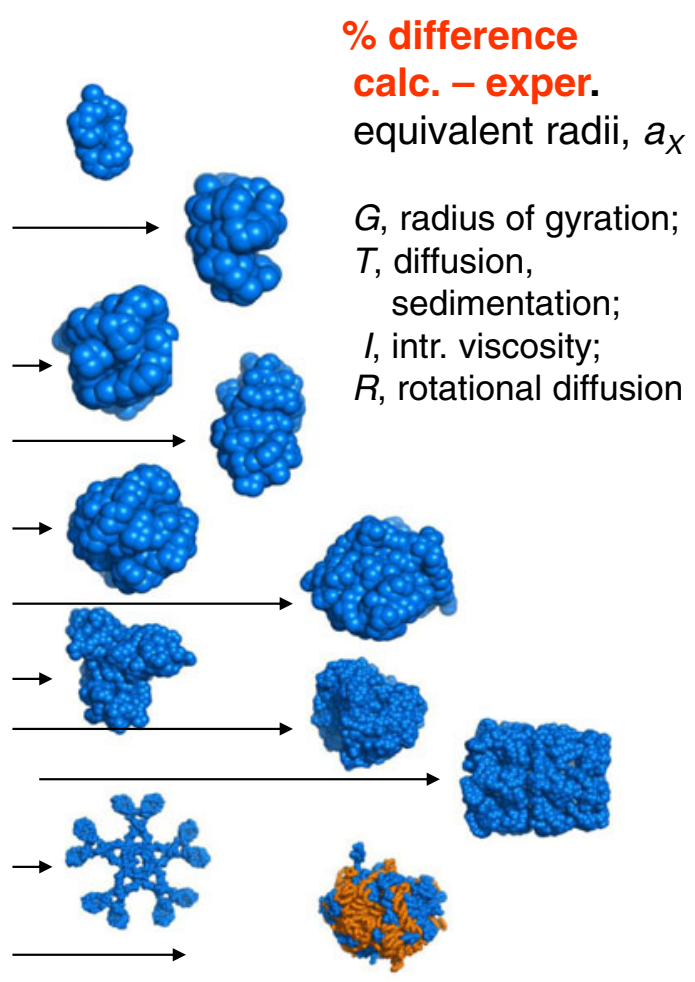

Fig. 3 Numerical results for the predicted solution properties of some rigid proteins, as obtained from the three procedures, with indication of the deviation of the calculated results from the experimental ones 


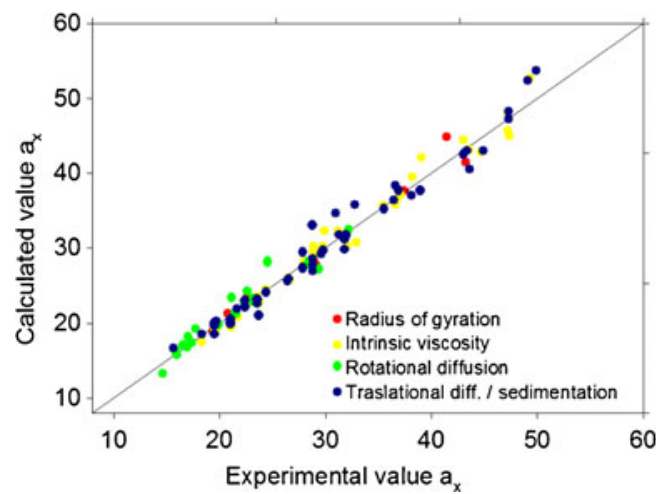

Fig. 4 Plot of calculated versus experimental values of the equivalent radii, for the Residue/PHM method, showing the predictive capability of this scheme. Over 150 data from the various solution properties are displayed in the plot

In its simplest mode of interpretation, dynamic NMR spectroscopy provides an overall correlation time, $\tau_{c}$, which corresponds to the harmonic mean relaxation time of the rigid body, $\tau_{\mathrm{h}}$. However, the amount of information that can be extracted from this technique is much more abundant.

For each amino acid residue in the protein, several quantities can be extracted, and within a dynamics perspective, the most important ones are the longitudinal and transverse relaxation times, $T_{1}$ and $T_{2}$. These quantities are hydrodynamic properties, which depend primarily on the rotational diffusion tensor of the protein as a whole, but their distinct values for each residue depend also on the orientation of their $\mathrm{N}-\mathrm{H}$ or $\mathrm{C}^{\alpha}-\mathrm{H}$ bonds. Thus, the series of NMR relaxation times contains information not only on the overall size and shape of the rigid protein but also on the placement of each residue within its internal structure.

After the proven ability of the HYDROPRO scheme for predicting the overall hydrodynamics of protein structures had been established - particularly the rigid-body rotational diffusion tensor-HYDRONMR was then developed. Essentially, the development consisted of the detection of the orientation of the involved bond vectors for each residue, and the calculation of $T_{1}, T_{2}$ and other properties using existing theory to predict them from rotational diffusivity and bond orientation - see, for instance, Palmer et al. (1996) and Tjandra et al. (1997). An example of the experimental results and HYDRONMR predictions is presented in Fig. 5.

\section{Solution properties from lower-resolution structure}

Other structural techniques, like cryo-electron microscopy (cryoEM) and small-angle, x-ray or neutron scattering (SAS), yield structures with a resolution lower than the atomic- or residue-level structures provided by other methods. Nonetheless, those lower-resolution structures are still sufficient for reliable prediction of solution properties, and tools for making such predictions are available.

The outcome of cryoEM is a 3D density map from which the particle's reconstruction is obtained from the points with density above a given threshold. HYDROMIC (Garcia de la Torre et al. 2001) reads the density map and builds hydrodynamic shell model from which the solution properties can be derived. Figure 6 displays the cryoEM-derived contour and its corresponding shell model. For some recent illustrative examples of HYDROMIC applications, see, for instance, Niewiarowski et al. (2010), Braun et al. (2011) or Del Castillo et al. (2011).

Other low-resolution techniques are small-angle neutron and X-ray scattering (acronyms SANS and SAXS, respectively). These techniques, particularly SAXS, are particularly used to obtain estimates of the size and shape of the particle from the angular dependence of the scattering intensity. The widely employed analysis programs, developed by Svergun and coworkers, including from DAMMIN (Svergun 1999) to DAMMIF (Franke and Svergun 2009) yield a 3D reconstruction in the form of an array of "pseudoatoms" that represent the size and shape of the macromolecule. Then, a number of authors have employed the first
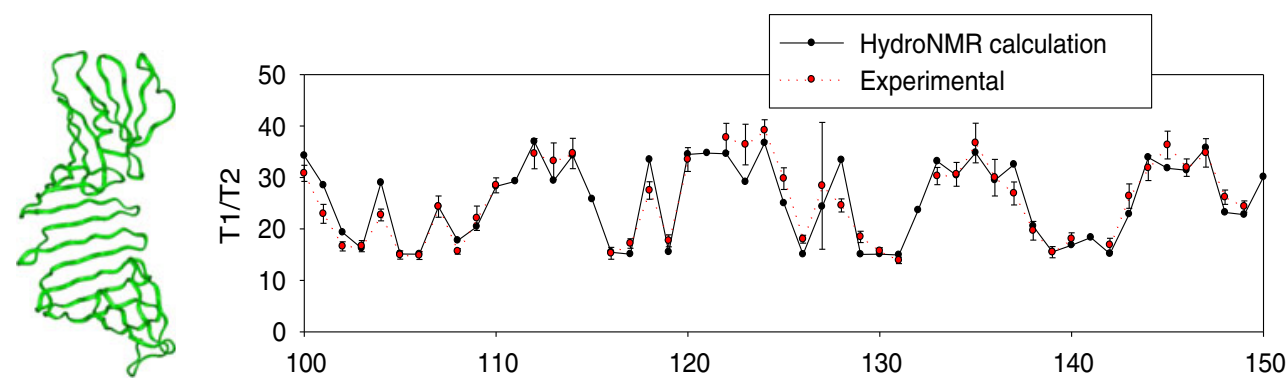

Fig. 5 NMR Relaxation times of outer surface protein A, a highly anisotropic protein with 272 residues. The HYDROPRO calculation in the Atomic/PHM $\rightarrow$ Shell mode predicts a $\tau_{\mathrm{c}}$ of $13.3 \mathrm{~ns}$ with the standard choice $a=2.9 \AA$, in very good agreement with the experimental value of

$13.7 \mathrm{~ns}$. But this single value is not the only one that is nearly matched by the prediction; the HYDRONMR results for the series of $T_{1} / T_{2}$ ratios for the successive residues reproduce extremely well the experimental data (values shown for the region 100-150) 

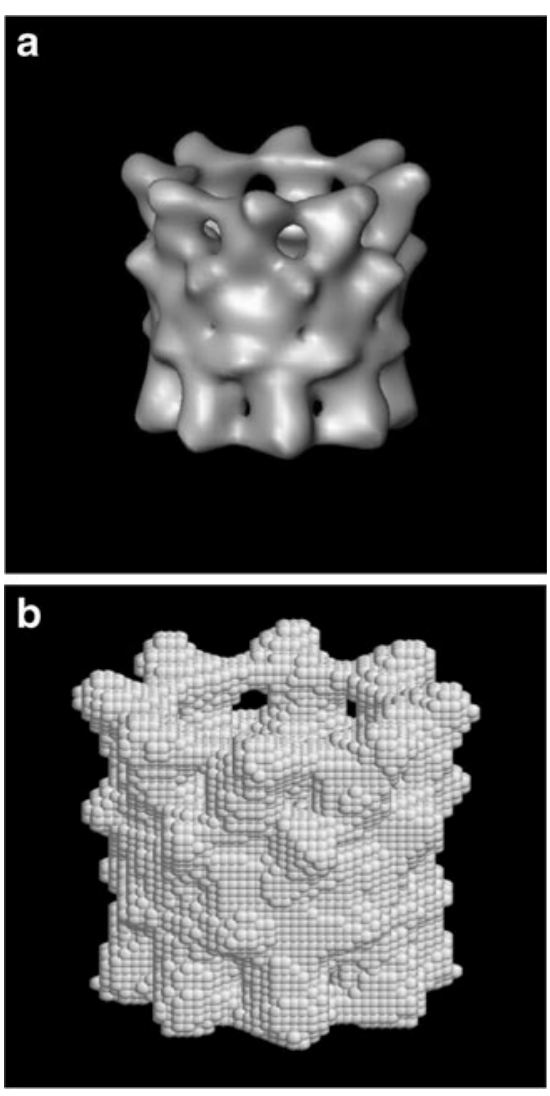

Fig. 6 a The $3 \mathrm{D}$ reconstruction of cytosolic CCT chaperonin, a large protein complex with molecular weight nearly $1,000 \mathrm{kDa}$. b Shell model constructed by HYDROMIC and used in the calculation of the sedimentation coefficient, with a result $\mathrm{s}_{20, w}=24.1 \mathrm{~S}$, in close agreement with the experimental value 25.6 S. For details, see Garcia de la Torre et al. (2001)

version of HYDROPRO (Garcia de la Torre et al. 2000) to predict the hydrodynamic properties.

As examples of successful applications, see Scott et al. (2002), Baldock et al. (2011), and Patel et al. (2012). In these applications of the Atomic/PHM $\rightarrow$ Shell mode HYDROPRO to models which do not contain true atoms, the authors made reasonable, ad hoc estimations of the value of the pseudo-atom radius, $a$, to be employed in the calculations. At the time of writing this, we are devising an automated procedure, HYDROSAS, which, as in the case of HYDROMIC for cryoEM, would take the pseudo-atom model directly derived from the small-angle scattering (SAS) data by the reconstruction program, optimise internally the value of $a$, and yield the final values of the hydrodynamic properties.

\section{Solution properties from subunit-level structure}

There may be instances in which the available level of structural detail is even lower than in the above-described applications, so that the hydrodynamic model must be accordingly simple. It may also happen that, although high-level structural information would be available, one would wish to concentrate on a few essential aspects of the structure, so that the level of description of other details is unimportant.

In such cases, the macromolecule can be regarded as composed of a few subunits, which can indeed correspond to well-defined domains or entities, but may also be simply model building blocks. This is the basis for the methodology implemented in the computer program HYDROSUB (Garcia de la Torre and Carrasco 2002), in which the primary model is an array of subunits having the shape of cylinders (rods or disks) or ellipsoids of revolution. The structure is specified by a list, containing one register for each subunit, giving the sizes (axis lengths, or length and diameter), position of its centre, and orientation of the main axis. Internally, HYDROSUB constructs a shell model for which the properties are calculated.

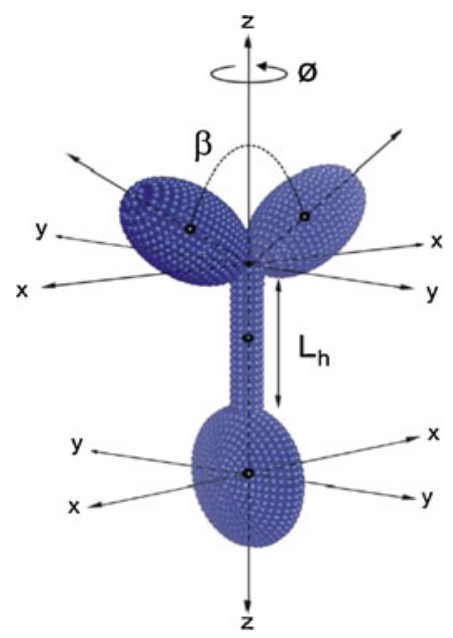

Fig. 7 Modelling the structure of IgG3 wild-type (WT) and mutant (M15) antibodies. a General multisubunit model for IgG antibodies employed in the HYDROSUB calculations. b, c For the WT and M15
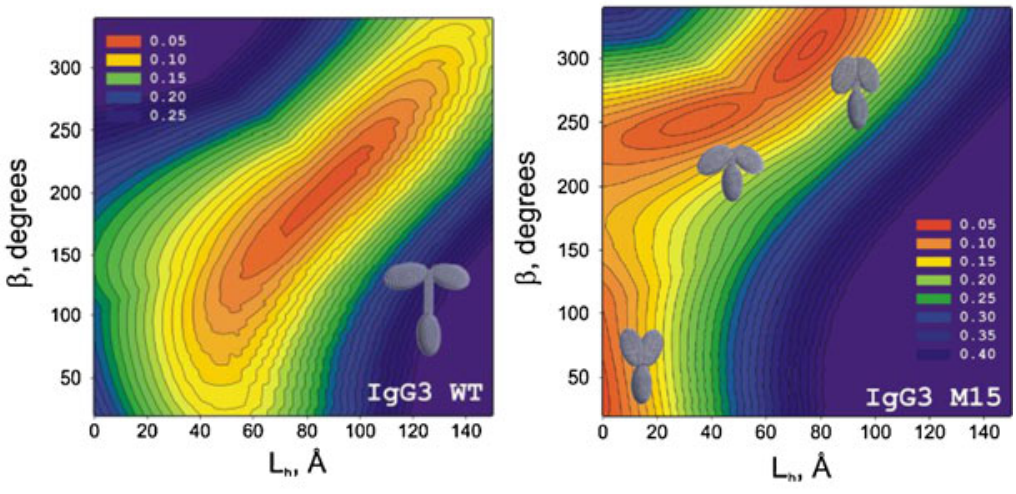

antibodies, contour plot of the values of target function, $\Delta$, as a function of the angle of between the two Fab subunits and the hinge length 
The paradigmatic application of this procedure is the determination of the basic structural features of antibody molecules. For instance, antibody IgG consists of three nearly globular subunits - two Fabs and one Fc - joined by a connector. The size and shape of the subunits is nearly the same, regardless of the specific origin or class of the IgG molecule. The subunits may be isolated and independently characterised by measuring solution properties; more recently, it has been possible to determine their detailed structure from x-ray diffraction, so that, alternatively, the solution properties can be predicted by HYDROPRO. Then, from the solution properties, the dimensions of the equivalent ellipsoids can be determined as described, the surfaces represented by bead-shell models which are then assembled via a linking hinge region to give a structure representing the average domain orientation of an intact immunologically active antibody (Carrasco et al. 1999, 2001; Lu et al. 2006, 2007).

Thus, even if one could construct full atomic models from the atomic structures of the subunits, the HYDROSUB model always allows the calculation of the solution properties of such multisubunit structures. In the case of IgG antibodies, essential features are the hinge length and the disposition of the arms. Models constructed with ellipsoids for the subunits and a rod for the hinge allows one to concentrate on the dependence of solution properties on these aspects. Figure 7 illustrates the structural elucidation that differentiated the wild-type and a mutant form of the IgG3 antibody (Amoros et al. 2010). Having determined for each form a large set of experimental properties, namely the sedimentation coefficient and intrinsic viscosity, as well as the radius of gyration and longest distance from SAXS, the HYDROSUB calculations for multiple structures were analysed by means of HYDROFIT. For the wild-type, a unique, best-fitting structure is found, whose salient feature is a long hinge, with the calculated values of all the properties in excellent agreement with the experimental ones. For the mutant, several similarly well-fitting structures are found, all having in common an extremely short hinge, although several arrangements of the arms were compatible with the solution properties. This illustrates how the combined HYDROSUB/HYDROFIT treatment may determine the main structural features of multisubunit structures from solution properties, providing numerical values of the essential parameters, and informing on quality and uniqueness of the structural determination.

The HYDROSUB approach has been successfully employed in other, quite different, applications; see, for instance, Zorrila et al. (2007), Gapinski et al. (2010) or Sokolova et al. (2010).

\section{Concluding comment}

The programs in the ELLIPS suite can be downloaded from the NCMH website http://www.nottingham.ac.uk/ncmh complete with full instructions. In any publication, users are requested to acknowledge Salford Software and the Numerical Algorithms Group, Oxford, for ELLIPS2-4, and BUSS Limited for use of SimplePlot Library routines in ELLIPS3.

The programs in the HYDRO suite can be downloaded from http://leonardo.inf.um.es/macromol/ along with detailed documentation (user guides and sample files).

Acknowledgment The algorithms we have reviewed here are the culmination of the efforts of a considerable number of colleagues and we would like to express our deep gratitude to them. We would also like to congratulate our good friend and colleague Professor Allen Minton on his 70th birthday and appreciate greatly all that he has done to advance our knowledge of the way biological macromolecules behave in a solution environment - the environment in which many occur naturally. In particular, we acknowledge the great contribution he has made to our understanding of the way macromolecules behave in a crowded or non-ideal environment. Although in this review we have described conditions in which we try to avoid or minimise these effects, in some cases non-ideality behaviour can yield very useful information about conformation - as we have considered in our article. Professor Minton has also always supported the use of dilute-solution techniques for macromolecular characterisation, and in many occasions he has stimulated the development of advances in hydrodynamic modelling.

Support to J.G.T. was provided by grant CTQ-2009-06831 from the Ministerio de Ciencia e Innovacion including FEDER funds. Final revision and publication in 2013 was under grant CTQ201233717 from the Ministerio de Ciencia y Competitividad". Support to J.G.T was also provided by Fundación Séneca. Work by S.E.H. was funded by the Biotechnology and Biological Sciences Research Council (grant BBD01364X1), the Knowledge Transfer Network (KTN SPARK award) and partially by the Royal Society (grant RFBR JP101601).

Conflict of interest statement There are no conflicts of interest.

Open Access This article is distributed under the terms of the Creative Commons Attribution License which permits any use, distribution, and reproduction in any medium, provided the original author(s) and the source are credited.

\section{References}

Abdelhameed AS, Morris G, Adams GG, Rowe AJ, Laloux O, Cerny L, Bonnier B, Duvivier P, Conrath K, Lenfant C, Harding SE (2012) An asymmetric and slightly dimerized structure for the tetanus toxoid protein used in glycoconjugate vaccines. Carbohyd Polym 90:1831-1835

Amoros D, Ortega A, Harding SE, García de la Torre J (2010) Multi-scale calculation and global-fit analysis of hydrodynamic properties of biological macromolecules: determination of the overall conformation of antibody IgG molecules. Eur Biophys J 39:361-370

Ang S, Kogulanathan J, Morris GA, Kök MS, Shewry PR, Tatham AS, Adams GG, Rowe AJ, Harding SE (2010) Structure and heterogeneity of gliadin: a hydrodynamic evaluation. Eur Biophys J 39:255-261

Aragon S, Hahn DK (2006) Precise boundary element computation of proteins transport properties: diffusion tensors, specific volume and hydration. Biophys J 91:1591-1603 
Baldock C, Oberhauser AF, Ma L, Lammie D, Siegler V, Mithieux SM, Tu Y, Chow JY, Suleman F, Malfois M, Rogers S, Guo L, Irving TC, Wess TJ, Weiss AS (2011) Shape of tropoelastin, the highly extensible protein that controls human tissue elasticity. Proc Natl Acad Sci USA 108:4322-4327

Bernado P, Blackledge M (2009) A self-consistent description of the conformational behavior of chemically denaturated proteins from NMR and small angle scattering. Biophys J 97:2839-2845

Bloomfield VA, Dalton KO, Van Holde KE (1967) Frictional coefficients of multisubunit structures. I. Theor Biopolym 5:135-148

Brady RL, Edwards DJ, Hubbard RE, Jiang JS, Lange G, Roberts SM, Todd RJ, Adair JR, Emtage JS, King DJ, Low DC (1992) Crystal structure of a chimaeric Fab' fragment of an antibody binding tumour cells. J Mol Biol 227:253-264

Braun N, Zacharias M, Peschek J, Kastenmüller A, Zou J, Hanzlik M, Haslbeck M, Rappsilber J, Weinkauf S (2011) Multiple molecular architectures of the eye lens chaperone $\alpha \beta$-crystallin elucidated by a triple hybrid approach. Proc Natl Acad Sci USA 108:2049120496

Brookes E, Demeler B, Rocco O (2010) Developments in the USSOMO bead modelling suite: new features in the residue-to-bead, improved grid routines and influence of solvent accessible area screening. Macromol Biosci 10:746-753

Byron O (1997) Constructions of hydrodynamic bead models from high-resolution x-ray crystallographic or nuclear magnetic resonance data. Biophys J 72:408-415

Carrasco B, Garcia de la Torre J (1999) Hydrodynamic properties of rigid particles. Comparison of different modelling and computational procedures. Biophys J 76:3044-3057

Carrasco B, Garcia de la Torre J, Byron O, King D, Walters C, Jones S, Harding SE (1999) Novel Size-Independent Modelling of Dilute Solution Conformation of the Immunoglobulin IgG Fab' Domain Using SOLPRO and ELLIPS. Biophys J 77:2902-2910

Carrasco B, Garcia de la Torre J, Davis KG, Jones S, Athwal D, Walters C, Burton DR, Harding SE (2001) Crystallohydrodynamics for solving the hydration problem for multi-domain proteins: open physiological conformations for human IgG. Biophys Chem 93:181-196

Cho M, Noder G, Kim H, Jensen MR, Bernado P, Fernandez CO, Becker M, Blackledge M, Zweckstetter M (2009) Structural characterization of the $\alpha$-synuclein in an aggregation prone state. Protein Sci 18:1840-1846

Del Castillo U, Alfonso C, Acebron SP, Martos A, Moro F, Rivas G, Muga A (2011) A quantitative analysis of the effect of nucleotides and the $\mathrm{M}$ domain on the association equilibrium of $\mathrm{ClpB}$. Biochemistry 50:1991-2003

Denisov VP, Halle B (1996) Protein hydration dynamics in aqueous solution. Faraday Discuss 103:245-253

Franke D, Svergun DI (2009) DAMMIF, a program for rapid ab-initio shape determination in small-angle scattering. J Appl Crystallogr 42:342-346

Gapinski J, Szymanski J, Wilk A, Kohlbrecher J, Patkowski A, Holyst R (2010) Size and Shape of Micelles Studied by Means of SANS, PCS, and FCS. Langmuir 26:9304-9314

Garcia de la Torre J, Bloomfield VA (1981) Hydrodynamic properties of complex, rigid, biological macromolecules, theory and applications. Q Rev Biophys 14:81-139

Garcia de la Torre J, Carrasco B (2002) Hydrodynamic Properties of Rigid Macromolecules Composed of Ellipsoidal and Cylindrical Subunits. Biopolymers 63:163-167

García de la Torre J, Navarro S, Lopez Martinez MC, Diaz FG, Lopez Cascales JJ (1994) HYDRO: a computer software for the prediction of hydrodynamic properties of macromolecules. Biophys J 67:530-531

García de la Torre J, Huertas ML, Carrasco B (2000) Calculation of hydrodynamic properties of globular proteins from their atomiclevel structures. Biophys J 78:719-730
García de la Torre J, Llorca O, Carrascosa JL, Valpuesta JM (2001) HYDROMIC: Prediction of hydrodynamic properties of rigid macromolecular structures obtained from electron microscopy. Eur Biophys J 30:457-462

Harding SE (1981) A compound hydrodynamic shape function derived from viscosity and molecular covolume measurements. Int J Biol Macromol 3:340-341

Harding SE (1983) Triaxial ellipsoids as models for macromolecules in solution; procedures for numerical inversion of the shape functions leading to a stable unique solution. Comput Biol Med 13:89-97

Harding SE (1987) A General Method for Modelling Macromolecular Shape in Solution. A Graphical (П - G) Intersection Procedure for Triaxial Ellipsoids. Biophys J 51:673-680

Harding SE (1995) On the hydrodynamic analysis of macromolecular conformation. Biophys Chem 55:69-93

Harding SE (1997) The intrinsic viscosity of biological macromolecules. Progress in measurement, interpretation and application to structure in dilute solution. Prog Biophys Mol Biol 68:207-262

Harding SE, Dampier M, Rowe AJ (1982) The viscosity increment for ellipsoids of revolution: Some observations on the Simha formula. Biophys Chem 15:205-208

Harding SE, Horton JC, Cölfen H (1997) The ELLIPS suite of macromolecular conformation algorithms. Eur Biophys J 25:347-359

Harding SE, Cölfen H, Aziz Z (2005) The ELLIPS suite of whole-body protein conformation algorithms for Microsoft Windows. In: Scott D, Harding SE, Rowe AJ (eds) Analytical Ultracentrifugation. Techniques and Methods. Royal Society of Chemistry, Cambridge, pp 468-483

Krzeminski M, Marsh J, Naele C, Choy W-Y, Forman-Kay (2013) Characterization of disordered proteins with ENSEMBLE. Bioinformatics (in press)

Longman E, Kreusel K, Tendler SB, Fiebrig I, King K, Adair J, O’Shea P, Ortega A, Garcia de la Torre J, Harding SE (2003) Eur Biophys J 32:503

Lu Y, Longman E, Davis K, Ortega A, Grossmann JG, Michaelsen TE, García de la Torre J, Harding SE (2006) Crystallohydrodynamics of Protein Assemblies: Combining Sedimentation, Viscometry, and X-Ray Scattering. Biophys J 91:1688-1697

Lu Y, Harding SE, Michaelsen TE, Longman E, Davis KG, Ortega A, Grossmann JG, Sandlie I, Garcia de la Torre J (2007) Solution conformation of wild type and mutant IgG3 and IgG4 immunoglobulins using Crystallohydrodynamics: possible implications for complement activation. Biophys J 93:3733-3744

Mehl JW, Oncley JL, Simha R (1940) Viscosity and the shape of protein molecules. Science 92:132-133

Niewiarowski A, Bradley AS, Gor J, McKay AR, Perkins SJ, Tsaneva IR (2010) Oligomeric assembly and interactions within the human RuvB-like RuvBL1 and RuvBLS complexes. Biochem J 429:113-125

Oncley JL (1940) Electric moments and relaxation times of protein molecules. J Phys Chem 44:1103-1113

Ortega A, García de la Torre J (2007) Equivalent radii and ratios of radii from solution properties as indicators of macromolecular shape, conformation and flexibility. Biomacromolecules 8:2464-2475

Ortega A, Amoros D, Garcia de la Torre J (2011a) Prediction of hydrodynamic and other solution properties of proteins from atomic- and residue-level models. Biophys J 101:892-898

Ortega A, Amoros D, García de la Torre J (2011b) Global fit and structure optimization of flexible and rigid macromolecules and nanoparticles from analytical ultracentrifugation and other dilute solution properties. Methods 54:115-123

Palmer AG, Williams J, McDermott A (1996) Nuclear magnetic resonance studies of biopolymer structures. J Phys Chem 100:13293-13310

Patel TR, Reuten R, Xion S, Meier M, Winzor DJ, Koch M, Stetefeld J (2012) Nano-structure of the laminin $\gamma-1$ short arm reveals an extended and curved domain. Matrix Biol 31:135-140 
Perrin F (1934) Movement Brownien d'un ellipsoide (I). Dispesion dielectrique pour des molecules ellipsoidales. J Phys Radium 5:497-511

Perrin F (1936) Mouvement Brownien d'un ellipsoide (II).Rotation libre et depolarisation des fluorescences. Translation et diffusion de molecules ellipsoidales. J Phys Radium 7:1-11

Rai N, Nollman M, Spotorno B, Tassara G, Byron O, Rocco M (2005) SOMO (Solution Modeler): differences between X-ray and NMRderived bead models suggest a role for side chain flexibility in protein hydration. Structure 13:723-734

Rallison JM, Harding SE (1985) Excluded volumes for pairs of triaxial ellipsoids at dominant Brownian motion. J Coll Int Sci 103:284-289

Saito N (1951) The effect of the Brownian motion on the viscosity of solutions of macromolecules. I. Ellipsoid of Revolution. J Phys Soc (Japan) 6:297-301

Scheraga HA, Mandelkern L (1953) Consideration of hydrodynamic properties of proteins. J Am Chem Soc 75:179-184

Scott DJ, Grossman JG, Tame JRH, Byron O, Wilson KS, Otto BR (2002) Low resolution solution structure of the apo form of Escherichia Coli haemoglobin protease Hbp. J Mol Biol 315:1179-1187

Simha R (1940) The influence of brownian movement on the viscosity of solutions. J Phys Chem 44:25-34
Sokolova A, Kealley C, Hanley T, Rekas A, Gilbert EP (2010) Smallangle $\mathrm{x}$-ray scattering study of the effect of $\mathrm{pH}$ and salts on $11 \mathrm{~S}$ soy glycinin in the freeze-dried powder and solution states. J Agric Food Sci 58:967-974

Svergun DI (1999) Restoring low resolution structure of biological macromolecules from solution scattering using simulated annealing. Biophys J 76:2879-2886

Tanford C (1961) Physical Chemistry of Macromolecules Chap. 4. Wiley, New York

Taylor WR, Thornton JM, Turnell WG (1983) An ellipsoidal approximation of protein shape. J Mol Graphics 1:30-38

Teller DC, Swanson E, De Haen C (1976) The translational friction coefficient of proteins. Meth Enzymol 61:103-124

Tjandra N, Garret DS, Gronenborn A, Bax A, Clore GM (1997) Defining long range order in NMR structure determination from the dependence of heteronuclear relaxation on rotational diffusion anisotropy. Nat Struct Biol 4:443-449

Zorrilla S, Doan T, Alfonso C, Margeat E, Ortega A, Rivas G, Aymerich S, Royer CA, Declerck N (2007) Inducer-modulated cooperative binding of the tetrameric $\mathrm{CggR}$ repressor to operator DNA. Biophys J 92:3215-3227 